\title{
PROPER GROUP ACTIONS AND SYMPLECTIC STRATIFIED SPACES
}

\author{
L. BAtes AND E. LERMAN
}

\begin{abstract}
Let $(M, \omega)$ be a Hamiltonian $G$-space with a momentum map $F: M \rightarrow \mathbf{g}^{*}$. It is well-known that if $\alpha$ is a regular value of $F$ and $G$ acts freely and properly on the level set $F^{-1}(G \cdot \alpha)$, then the reduced space $M_{\alpha}:=F^{-1}(G \cdot \alpha) / G$ is a symplectic manifold. We show that if the regularity assumptions are dropped the space $M_{\alpha}$ is a union of symplectic manifolds, and that the symplectic manifolds fit together in a nice way. In other words the reduced space is a symplectic stratified space. This extends results known for the Hamiltonian action of compact groups.
\end{abstract}

\section{Introduction.}

Reduction of the number of degrees of freedom of a symplectic Hamiltonian system has a long history. The modern formulation of reduction is due to Meyer $[\mathrm{Me}]$ and to Marsden and Weinstein $[\mathrm{MW}]$. We recall their result. One starts with a symplectic manifold $(M, \omega)$, a Hamiltonian action of a Lie group $G$ and a corresponding equivariant momentum map $F: M \rightarrow \mathrm{g}^{*}$. Let $\mathcal{O}$ be a coadjoint orbit of $G$. If the momentum map is transversal to the orbit, then the preimage $F^{-1}(\mathcal{O})$ of the orbit is a submanifold of $M$ and the action of the Lie group $G$ on the preimage is locally free. Assume that this action is actually free and that the orbit map $F^{-1}(\mathcal{O}) \rightarrow F^{-1}(\mathcal{O}) / G$ is a fibration. The reduction theorem says that the orbit space $M_{\mathcal{O}}:=F^{-1}(\mathcal{O}) / G$ is a symplectic manifold. The restriction of a smooth $G$ invariant function $h$ on $M$ to the preimage of the orbit descends to a smooth function $h_{\mathcal{O}}$ on the reduced space $M_{\mathcal{O}}$. Moreother, the Hamiltonian flow of $h$ on $F^{-1}(\mathcal{O})$ descends to the Hamiltonian flow of $h_{\mathcal{O}}$ on the reduced space.

It turns out that often the action is only locally free, so at best the reduced spaces are symplectic orbifolds. This already suggests that the category of symplectic manifolds is too restrictive for Hamiltonian dynamics. More generally one would like to get rid of the transversality hypothesis in the reduction procedure. One reason for this desire is that the more symmetry the point of a system has the more singular the momentum map is at this point. Of course, the symmetric points are not generic, but they are very 
important in understanding of the dynamics of the system. Another reason is that one would like to understand the change in the topology of the reduced space as one crosses the critical values of the momentum map.

For a number of years the reduction at singular values of the momentum map has been problematic. In 1981 Arms, Marsden and Moncrief [AMM] showed that under some assumptions the set $F^{-1}(0) / G$ is a union of symplectic manifolds and that the flow of invariant Hamiltonians on the level set $F^{-1}(0)$ of the momentum map descends to the flow of the reduced Hamiltonians on these symplectic manifolds. Otto showed that a similar result holds for arbitrary coadjoint orbits, i.e., that $F^{-1}(\mathcal{O}) / G$ is a union of symplectic orbifolds $[\mathbf{O}]$. Yet these observations didn't gain use and are not well known. Many reduction schemes have been proposed since then. A number of them are compared in $[\mathbf{A G J}]$.

Our approach to reduction is the one proposed in $[\mathbf{S L}]$ and [LMS]. Namely, for a point $\alpha$ in the dual of Lie algebra of $G$, the reduced space at $\alpha$ is the topological space $M_{\alpha}=M_{G \cdot \alpha}:=F^{-1}(G \cdot \alpha) / G$, where $G \cdot \alpha$ is the coadjoint orbit through $\alpha$. In general this topological space can be quite horrible, as we shall see shortly. One of the main points of the paper is that we only need to make two assumptions - that the action is proper and that the coadjoint orbits of our group are locally closed - to guarantee that the reduced spaces are manageable. By 'manageable' we mean that Hamilton's equations hold and the geometry of the reduced space is reflected in the dynamics.

We will also show that in analogy with symplectic orbifolds (which are modeled on a symplectic vector space modulo a finite group) our reduced spaces are modeled on symplectic vector spaces reduced at zero with respect to a linear action of a compact group. This extends the results of [SL] and $[\mathrm{CS}]$ which proved the above assertions for the case of the compact symmetry group. One motivation for the extension is to push the methods of [SL] as far as they would go. Another motivation for this extension comes from field theory, where the symmetry groups are not compact. Yet some field theories such as Yang-Mills in bounded domains do not satisfy the assumptions of the Arms-Marsden-Moncrief theory (for which field theory appears to be a primary motivation), but the gauge group still acts properly, and a large portion of the finite dimensional results can still be established [SSB].

We now briefly describe the organization of the paper.

1. We start by defining an algebra of "smooth functions" on the reduced space with a natural Poisson bracket. The bracket allows us to define Hamiltonian flows of smooth functions on the reduced space. If the smooth functions on the reduced space separate points the flows are unique.

2. The Hamiltonian flows of smooth functions preserve the decomposition 
of the reduced space induced by the orbit type decomposition of the original manifold.

3. Local normal form computations show that

(a) the orbit type decomposition of the reduced space is a decomposition into symplectic manifolds;

(b) the embeddings of these manifolds (the symplectic pieces) into the reduced space are Poisson maps;

(c) the group generated by the Hamiltonian flows of functions on the reduced space acts transitively on the connected components of the symplectic pieces;

(d) consequently, the Poisson algebra of smooth functions on the reduced space carries all the information about the decomposition of the reduced space into symplectic pieces.

4. The last fact allows us to define isomorphisms of reduced spaces in terms of the corresponding isomorphisms of Poisson algebras of functions. We can also define local isomorphisms.

5. Local normal form computations show that a reduced space is locally isomorphic to a symplectic vector space reduced at zero with respect to a linear action of a compact group. This symplectic vector space is the maximal symplectic subspace of the slice to the corresponding orbit in the original manifold.

6. It follows that the decomposition of the reduced space by orbit type is a stratification and that the local structure of a stratification can be read off from the slice representation.

7. We use the local normal form computation to show that the strata of the reduced space can individually be obtained by Marsden-WeinsteinMeyer reduction. This provides us with a way to reconstruct the original dynamics from the dynamics on the reduced space.

8. We conclude by showing how one can use symplectic cross-sections to factor out the coadjoint orbit directions.

\section{Dynamics on the reduced space.}

Consider a symplectic manifold $M$ with a Hamiltonian action of a Lie group $G$ and let $F: M \rightarrow \mathrm{g}^{*}$ be a corresponding equivariant momentum map. Fix a coadjoint orbit $\mathcal{O}$ of $G$. We define the corresponding reduced space $M_{\mathcal{O}}$ to be the topological quotient of the subset $F^{-1}(\mathcal{O})$ of $M$ by the action of the group $G$,

$$
M_{\mathcal{O}}:=F^{-1}(\mathcal{O}) / G .
$$


We have not made enough assumptions to guarantee that the set $F^{-1}(\mathcal{O})$ is a manifold or that the quotient space $M_{\mathcal{O}}$ is nice.

Example 1. Consider an irrational flow on a torus $\mathbf{R} \times \mathbf{T} \rightarrow \mathbf{T}^{2}$ generated by a vector $\xi$ in the Lie algebra of $\mathbf{T}^{2}$. The flow lifts to a Hamiltonian action on the cotangent bundle of the two torus. The reduced space at zero $M_{0}$ is homeomorphic to $\mathbf{T} / \mathbf{R} \times \mathbf{R} \xi^{\circ}$ where $\mathbf{R} \xi^{\circ}$ is the annihilator in the dual of the Lie algebra of the torus of the line through $\xi$. The reduced space is not Hausdorff.

We note for future reference that the space of functions on the cotangent bundle of the two torus that are invariant under the flow is isomorphic to the space of functions on $\mathbf{R}^{2}$ and that the Poisson bracket of two invariant functions is zero.

Our first step in defining the dynamics on the reduced space (in this we are following $[\mathbf{A C G}]$ ) is to define a Poisson algebra of "smooth functions" on the reduced space. Since the restriction of a smooth invariant function on the manifold $M$ to the set $F^{-1}(\mathcal{O})$ descends to a continuous function on the quotient $F^{-1}(\mathcal{O}) / G=M_{\mathcal{O}}$, we define the smooth functions on the reduced space to be these restriction,

$$
\begin{aligned}
C^{\infty}\left(M_{\mathcal{O}}\right) & :=\left.C^{\infty}(M)^{G}\right|_{F^{-1}(\mathcal{O})} \\
& :=C^{\infty}(M)^{G} / \mathcal{I} .
\end{aligned}
$$

Here $C^{\infty}(M)^{G}$ is the algebra of smooth $G$-invariant functions on the manifold $M$, and $\mathcal{I}=\mathcal{I}\left(F^{-1}(\mathcal{O})\right)$ is the ideal of invariant functions that vanish on the set $F^{-1}(\mathcal{O})$.

To show that the algebra of smooth functions $C^{\infty}\left(M_{\mathcal{O}}\right)$ is a Poisson algebra, we need to check that $\mathcal{I}$ is not only an ideal under multiplication of functions but also an ideal with respect to the Poisson bracket (recall that the $G$ invariant functions form a Poisson subalgebra of $\left.C^{\infty}(M)\right)$. The fact that $\mathcal{I}$ is a Poisson ideal follows from Lemma 2 below.

Lemma 2. Let $M$ be a symplectic (or, more generally, a Poisson) manifold, and $\mathcal{A}$ be a Poisson subalgebra of $C^{\infty}(M)$. Suppose that the Hamiltonian flows of functions in $\mathcal{A}$ preserve a subset $X$ of the manifold $M$. Then the ideal

$$
\mathcal{I}(X):=\left\{f \in \mathcal{A}:\left.f\right|_{X}=0\right\}
$$

of functions in $\mathcal{A}$ that vanish on $X$ is a Poisson ideal of $\mathcal{A}$.

Proof. Let $f$ be in $\mathcal{A}, x$ be a point in $X$ and $h$ be in the ideal $\mathcal{I}(X)$. Let $\gamma(t)$ be the integral curve of the Hamiltonian vector field of $f$ with $\gamma(0)=x$. 
Then $\gamma(t)$ is in $X$ and so $h(\gamma(t))=0$ for all $t$. Differentiation with respect to $t$ yields

$$
0=\left.\frac{d}{d t}\right|_{0} h(\gamma(t))=\{f, h\}(x) .
$$

Thus $\left.\{f, h\}\right|_{X}=0$, i.e., $\{f, h\}$ is in the ideal $\mathcal{I}(X)$.

The Hamiltonian flows of invariant functions on $M$ preserve the fibers of the momentum map $F$ (Noether's theorem). Therefore, by Lemma 2 with $\mathcal{A}=C^{\infty}(M)^{G}$ and $X=F^{-1}(\mathcal{O})$ we have that $\mathcal{I}\left(F^{-1}(\mathcal{O})\right)$ is a Poisson ideal. This proves that the smooth functions $C^{\infty}\left(M_{\mathcal{O}}\right)$ on the reduced space form a Poisson algebra.

Remark. More generally, we can define a sheaf of Poisson algebras on the reduced space, a kind of structure sheaf. An open set $U$ in the reduced space $M_{\mathcal{O}}$ is the quotient of the intersection of the level set $F^{-1}(\mathcal{O})$ with a $G$ invariant open set $\tilde{U}$. We define the Poisson algebra $C^{\infty}(U)$ by

$$
C^{\infty}(U):=\left.C^{\infty}(\tilde{U})^{G}\right|_{F^{-1}(\mathcal{O})} .
$$

Remark. Recall that if the action of the group $G$ on the manifold $M$ is proper, then for a subgroup $H$ of $G$ the set of points $M_{(H)}$ of orbit type $H$, i.e., the set of points with orbits isomorphic to $G / H$, is a submanifold of $M$ (the definition of proper actions and some of their properties are listed later). Since the Hamiltonian flow of a $G$ invariant function on $M$ is $G$ equivariant, the manifolds $M_{(H)}, H<G$, are preserved by the flows of invariant functions. Therefore for a subgroup $H$ of $G$ the ideal of invariant functions vanishing on the intersection $F^{-1}(\mathcal{O}) \cap M^{(H)}$ is a Poisson ideal in the algebra of the invariant functions and consequently defines a Poisson ideal in the algebra of smooth functions $C^{\infty}\left(M_{\mathcal{O}}\right)$ on the reduced space.

The Poisson bracket on the reduced space should allow us to write down equations of motion for any $f \in C^{\infty}\left(M_{\mathcal{O}}\right)$. But first we need to define what we mean by a smooth curve in a reduced space.

Definition 3. A smooth curve $\gamma$ in a reduced space $M_{\mathcal{O}}$ is a continuous map $\gamma: I \rightarrow M_{\mathcal{O}}, I$ an interval, such that for any smooth function $h \in C^{\infty}\left(M_{\mathcal{O}}\right)$ the function $h(\gamma(t))$ is a smooth function on the interval $I$.

A smooth flow $\left\{\phi_{s}\right\}$ on $M_{\mathcal{O}}$ is defined similarly. It is a one-parameter group of homeomorphisms $\phi_{s}: M_{\mathcal{O}} \rightarrow M_{\mathcal{O}}$ such that for each $h \in C^{\infty}\left(M_{\mathcal{O}}\right)$ and each $s$, we have $h \circ \phi_{s} \in C^{\infty}\left(M_{\mathcal{O}}\right)$, and for each point $m \in M_{\mathcal{O}}$ the curve $s \mapsto \phi_{s}(m)$ is a smooth curve.

We are now in a position to define a Hamiltonian flow of a smooth function on a reduced space. 
Definition 4. A Hamiltonian flow of a smooth function $f$ on a reduced space $M_{\mathcal{O}}$ is a smooth flow $\left\{\phi_{s}\right\}$ such that for any point $m$ in $M_{\mathcal{O}}$ and any smooth function $h$ in $C^{\infty}\left(M_{\mathcal{O}}\right)$ we have

$$
\frac{d}{d s} h\left(\phi_{s}(m)\right)=\{f, h\}\left(\phi_{s}(m)\right)
$$

where $\{$,$\} is the Poisson bracket on C^{\infty}\left(M_{\mathcal{O}}\right)$.

This definition raises a problem. Since the reduced space $M_{\mathcal{O}}$ is not necessarily locally Euclidean, Equation (1) is not in general a system of ordinary differential equations in a coordinate-free notation. Therefore the existence and uniqueness of solutions of (1) needs to be addressed.

The existence is easy. The key fact that the Hamiltonian flow of a $G$ invariant function $\bar{f}$ on the original symplectic manifold $M$ is $G$ equivariant. Since the flow also preserves the level sets $F^{-1}(\mathcal{O})$ of the momentum map $F$, it descends to a flow on the reduced space $M_{\mathcal{O}}$. It is now a formal exercise to check that this flow is smooth, and that it is a Hamiltonian flow of the corresponding function $f \in C^{\infty}\left(M_{\mathcal{O}}\right)$ in the sense of the above definition (cf. p. 389 in $[\mathbf{S L}])$.

The uniqueness is not to be expected without additional assumptions about the topology of the reduced space. Indeed, on a non-Hausdorff manifold an integral curve of a vector field is not necessarily unique. One would expect non-uniqueness on any non-Hausdorff space. The example of the irrational flow on the cotangent bundle of the two torus considered above is quite instructive in this case. Recall that the reduced space at zero in the example is homeomorphic to the product $\left(\mathbf{T}^{2} / \mathbf{R}\right) \times \mathbf{R}$. It is easy to see that the smooth functions on this reduced space are simply the functions that are constant on the first factor and smooth (in the usual sense) on the second factor. It follows that any continuous flow on the product that fixes the points of the second factor is smooth. Since the induced Poisson bracket is zero, any flow that fixes the points of the second factor is a Hamiltonian flow of any smooth function. Thus a different set of ideas is needed to make sense of non-Hausdorff reduced spaces.

Lemma 5. If the smooth functions on the reduced space separate points, then Hamiltonian flows are unique.

Proof. Again we follow [SL], p. 389. Suppose that $\phi_{t}$ and $\psi_{t}$ are two Hamiltonian flows on a reduced space $M_{\mathcal{O}}$ generated by a function $f \in C^{\infty}\left(M_{\mathcal{O}}\right)$. Then, by the chain rule, $\phi_{-t}$ is a flow of $-f$. Since smooth functions separate points, it is enough to show that for any function $h \in C^{\infty}\left(M_{\mathcal{O}}\right)$ and any point $m$ in the reduced space,

$$
h\left(\psi_{t}\left(\phi_{-t}(m)\right)\right)=h(m) .
$$


However

$$
\frac{d}{d t} h\left(\psi_{t}\left(\phi_{-t}(m)\right)\right)=\{h, f\}\left(\psi_{t}\left(\phi_{-t}(m)\right)\right)+\{h,-f\}\left(\psi_{t}\left(\phi_{-t}(m)\right)\right)=0 .
$$

At this point we make an assumption that will guarantee that functions on the reduced space will separate points, namely that the action of the symmetry group $G$ on the original manifold $M$ is proper, that is to say the map

$$
G \times M \rightarrow M \times M, \quad(g, m) \mapsto(g \cdot m, m)
$$

is a proper map. Equivalently, an action of $G$ on $M$ is proper if given two convergent sequences $\left\{m_{n}\right\}$ and $\left\{g_{n} \cdot m_{n}\right\}$ in $M$ there exists a convergent subsequence $\left\{g_{n_{k}}\right\}$ in $G$.

\section{Digression: Properties of proper group actions.}

We now list the properties of proper group actions that we will need in the course of the paper. The proofs of some properties are easy or are readily available. Other properties appear to be folklore and we will supply the proofs in the appendix.

1. The isotropy group $G_{m}$ of any point $m$ in $M$ is compact; all orbits of $G$ in $M$ are closed and embedded submanifolds.

2. The orbit space $M / G$ is Hausdorff.

3. At every point $m \in M$ there exists a slice for the action of $G$. That is to say there is a ball $B$ about 0 in the fiber $T_{m} M / T_{m}(G \cdot m)$ of the normal bundle to the orbit through $m$ with $B$ invariant under the action of $G_{m}$ and an embedding $\phi: B \rightarrow M$ with $\phi(0)=m$ such that the set $G \cdot \phi(B)$ is open in $M$ and the induced map

$$
G \times_{G_{m}} B \rightarrow M, \quad[g, b] \mapsto g \cdot \phi(b)
$$

is a diffeomorphism onto the image $G \cdot \phi(B)$. Here $[g, b]$ denotes the class of $(g, b) \in G \times B$ in the associated fiber bundle $G \times_{G_{m}} B$ and $G_{m}$ is the isotropy group of $m$. The $G_{m}$ invariant manifold $\phi(B)$ is a slice for the action of $G$ at $m$.

4. There exists a $G$ invariant partition of unity subordinate to any $G$ invariant open cover. (We assume that the manifold $M$ is paracompact.)

5. There exists on $M$ a $G$ invariant positive definite metric.

6. Smooth $G$ invariant functions separate the orbits of $G$.

7. If $\omega$ is a $G$ invariant symplectic form on $M$ there exists a $G$ invariant almost complex structure $J$ adapted to $\omega$. That is to say, the bundle map $J$ is 
symplectic, $\omega(J \cdot, J \cdot)=\omega(\cdot, \cdot)$, and the symmetric form $\omega(\cdot, J \cdot)$ is a positive definite metric. A proof is provided in the appendix.

8. For any (compact) subgroup $H$ of $G$ the sets

$$
\begin{aligned}
M_{(H)} & =\left\{m \in M: G_{m}, \text { the isotropy group of } m, \text { is conjugate to } H\right\}, \\
M_{H} & =\left\{m \in M: G_{m} \text { is } H\right\},
\end{aligned}
$$

and

$$
M^{H}=\left\{m \in M: G_{m} \text { contains } H\right\}=\text { the set of points fixed by } H
$$

are submanifolds of $M$ (this follows from the existence of slices, cf. fact 3). The manifold $M_{(H)}$ is called the manifold of points of orbit type $(H)$. Note also that the closure of $M_{H}$ is contained in $M^{H}$ but need not equal all of $M^{H}$.

9. An equivariant version of the relative Darboux theorem holds:

Theorem 6 (Relative Darboux). Let $X$ be a submanifold of a manifold $Y$. Let $\omega_{0}$ and $\omega_{1}$ be two symplectic forms on $Y$ such that $\omega_{0}(x)=\omega_{1}(x)$ for each $x \in X$. Then there exist neighborhoods $\mathcal{U}_{0}$ and $\mathcal{U}_{1}$ of $X$ and a diffeomorphism $\psi: \mathcal{U}_{0} \longrightarrow \mathcal{U}_{1}$ such that the pull back of $\omega_{1}$ by $\psi$ is $\omega_{0}$ and $\psi$ is the identity on $X$.

If a Lie group $G$ acts properly on $Y$, preserves $X, \omega_{0}$ and $\omega_{1}$, then we can arrange that the neighborhoods $\mathcal{U}_{0}$ and $\mathcal{U}_{1}$ are $G$-invariant and that the diffeomorphism $\psi$ is $G$-equivariant.

A proof is given in the appendix.

10. It follows from fact 9 that if $M$ is symplectic then the manifolds $M_{H}$ and $M^{H}$ are symplectic as well. The manifold $M_{(H)}$ is usually not symplectic.

\section{Geometry of the reduced space.}

The main goal of this section is to establish the following theorem.

Theorem 7. Let $G$ be a Lie group acting properly and in a Hamiltonian way on a symplectic manifold $(M, \omega)$ with a corresponding equivariant momentum map $F: M \rightarrow \mathrm{g}^{*}$ and let $\mathcal{O}$ be a locally closed coadjoint orbit of $G$. Then:

(1) The reduced space $M_{\mathcal{O}}:=F^{-1}(\mathcal{O}) / G$ is a locally finite union of symplectic manifolds. We will call these manifolds symplectic pieces.

(2) The Hamiltonian flows of smooth functions preserve the decomposition of the reduced space $M_{\mathcal{O}}$ into symplectic pieces. 
(3) The embedding of a symplectic piece into the reduced space $M_{\mathcal{O}}$ is a Poisson map.

Observe that the condition of a coadjoint orbit being locally closed is automatic for reductive groups and for their semidirect products with vector spaces. There is an example of a solvable group due to Mautner ([P], p. 512) with non-locally closed coadjoint orbits so the condition we are imposing is not vacuous. Note also that the condition of the coadjoint orbit being locally closed is precisely the condition that is necessary in order for the shifting trick to make sense. Since we want to read off the structure of the reduced space from the corresponding slice representation on the original manifold we will not use the shifting trick.

Theorem 7 has an important corollary.

Corollary 8. Suppose $M_{\mathcal{O}}$ and $N_{\mathcal{O}^{\prime}}$ are two reduced spaces and $\phi: M_{\mathcal{O}} \rightarrow$ $N_{\mathcal{O}^{\prime}}$ a homeomorphism. If the induced pull-back map $\phi^{*} C^{\infty}\left(N_{\mathcal{O}}\right) \rightarrow C^{\infty}\left(M_{\mathcal{O}}\right)$ is a Poisson isomorphism then $\phi$ maps symplectic pieces to symplectic pieces.

Proof. On a connected symplectic manifold the group generated by the time one Hamiltonian flows of smooth functions acts transitively. It follows from this and from assertion (2) of the theorem that connected components of the symplectic pieces of a reduced space $M_{\mathcal{O}}$ are equivalence classes of the relation: $x$ is equivalent to $y$ if and only if there are smooth functions $f_{1}, \ldots, f_{n} \in C^{\infty}\left(M_{\mathcal{O}}\right)$ such that a composition of their time one flows maps $x$ to $y$. Thus the decomposition of a reduced space into symplectic manifolds is encoded in the Poisson algebra $C^{\infty}\left(M_{\mathcal{O}}\right)$ of smooth functions on the reduced space.

The corollary also allows us to define local isomorphisms of reduced spaces. We will see in Theorem 15 that all reduced spaces (under the two hypotheses above) are locally isomorphic to a symplectic vector space reduced at zero with respect to a linear action of a compact group. This in turn permits us to define abstract "symplectic stratified spaces."

Here is the strategy of the proof of Theorem 7. We will define the terms and provide complete statements shortly. Fix a point $x$ in the preimage $F^{-1}(\mathcal{O})$ of the coadjoint orbit. Since the symplectic form $\omega$ on $M$ is $G$ invariant the $G$ orbit of $x$ is a submanifold of constant rank. It follows by the constant rank embedding theorem of Marle [Ma2], [Ma1] (see Theorem 9 below) that a $G$ invariant neighborhood of the orbit $G \cdot x$ is symplectically determined by the restriction of the symplectic form to the orbit and by the symplectic normal bundle of the embedding $G \cdot x \hookrightarrow M$. (A non-equivariant version of the constant rank embedding theorem can also be found in [AG] on p. 26.) So if we can find a constant rank embedding of the orbit into 
some "simple" Hamiltonian $G$ manifold $Y$, a neighborhood of the orbit in $Y$ is going to be symplectically isomorphic to a neighborhood of the orbit in $M$. The manifold $Y$ is a kind of "Darboux coordinates" that take the action of $G$ into account. We will then carry out our computations of the reduced space in $Y$.

\section{Digression: Constant rank embeddings.}

Let $X$ be a submanifold of a symplectic manifold $(P, \tau)$. For a point $x$ in $X$ the symplectic perpendicular to the tangent space of $X$ at $x$ with respect to the form $\tau$ is the subspace

$$
T_{x} X^{\tau}:=\left\{v \in T_{x} P: \tau(x)(v, w)=0 \text { for all } w \in T_{x} X\right\} .
$$

Together these subspaces define a subbundle $T X^{\tau}$ of the restriction $T_{X} P$ of the tangent bundle of $P$ to $X$. Under the isomorphism

$$
T_{X} P \rightarrow T_{X}^{*} P \quad(x, v) \mapsto(x, \tau(x)(v, \cdot))
$$

the bundle $T X^{\tau}$ is identified with the annihilator $T X^{\circ}$ of $T X$ in $T_{X}^{*} P$. Since $T X^{\circ}=\left(T_{X} P / T X\right)^{*}$, the symplectic perpendicular $T X^{\tau}$ is isomorphic, as an abstract real vector bundle, to the normal bundle of $X$ in $P$. In general the form $\tau(x)$ may be degenerate on $T_{x} X^{\tau}$. In fact, the quotient $T_{x} X^{\tau} /\left(T_{x} X^{\tau} \cap\right.$ $\left.T_{x} X\right)$ is isomorphic to a maximal symplectic subspace of $\left(T_{x} X^{\tau}, \tau(x)\right)$. If the dimension of this quotient is constant, i.e., if the distribution $T X \cap T X^{\tau}$ is a vector bundle, we say that the embedding $X \hookrightarrow(P, \tau)$ is of constant rank. In this case the quotient bundle

$$
N(X):=T X^{\tau} / T X^{\tau} \cap T X
$$

is a symplectic vector bundle, the symplectic normal bundle of the embedding $X \hookrightarrow(P, \tau)$. Note that $T X \cap T X^{\tau}$ is simply the kernel of the restriction of $\tau$ to $X$ and that as abstract vector bundles

$$
T_{X} P \simeq N(X) \oplus\left(T X^{\tau} \cap T X\right) \oplus T X .
$$

So together the pull back $\left.\tau\right|_{X}$ and the symplectic normal bundle $N(X)$ contain more information than the abstract normal bundle of $X$ in $P$. In fact the two pieces of data $-\left.\tau\right|_{X}$ and $N(X)$ - uniquely describe the symplectic form $\tau$ in a whole neighborhood of $X$. The precise statement is this.

Theorem 9 (Uniqueness of constant rank embeddings). Let $(P, \tau)$ and $\left(P^{\prime}, \tau^{\prime}\right)$ be two symplectic manifolds. Suppose $i: X \rightarrow(P, \tau)$ and $i^{\prime}$ : $X \rightarrow\left(P^{\prime}, \tau^{\prime}\right)$ are two constant rank embeddings with isomorphic symplectic normal bundles such that $i^{*} \tau=\left(i^{\prime}\right)^{*} \tau^{\prime}$. Then there exist neighborhoods $U$ of 
$i(X)$ in $P$ and $U^{\prime}$ of $i^{\prime}(X)$ in $P^{\prime}$ and a diffeomorphism $\phi: U \rightarrow U^{\prime}$ such that $\phi \circ i=i^{\prime}$ and $\phi^{*} \tau^{\prime}=\tau$.

Furthermore, if $G$ is a Lie group that acts properly on $X, P$ and $P^{\prime}$, preserves the forms $\tau$ and $\tau^{\prime}$ and if the embeddings $i$ and $i^{\prime}$ are $G$ equivariant, then $U$ and $U^{\prime}$ can be chosen to be $G$ invariant and $\phi$ to be $G$ equivariant.

The proof of the theorem is given later in the appendix. Since any two equivariant momentum maps differ by a constant vector, we also have the following corollary (we keep the notation of Theorem 9).

Corollary 10. Suppose in addition that the actions of $G$ on $(P, \tau)$ and $\left(P^{\prime}, \tau^{\prime}\right)$ are Hamiltonian with momentum maps $F: P \rightarrow \mathrm{g}^{*}$ and $F^{\prime}: P^{\prime} \rightarrow \mathrm{g}^{*}$. If $F \circ i=F^{\prime} \circ i^{\prime}$ then $F^{\prime} \circ \phi=F$.

This ends our digression and we now continue with the proof of Theorem 7. Recall that we have a Hamiltonian $G$ space $\left(M, \omega_{M}\right)$ with momentum map $F: M \rightarrow \mathrm{g}^{*}$, that $x$ is a point in $M, \alpha=F(x)$ and $\mathcal{O}=G \cdot \alpha$ is the coadjoint orbit through $\alpha$. We want to model a neighborhood of the orbit $G \cdot x$ in $M$ in order to understand the structure of the quotient $F^{-1}(\mathcal{O}) / G$ near the orbit $G \cdot x$.

We have observed that $G \cdot x$ is a constant rank submanifold of $M$. We will now constract a symplectic manifold $\left(Y, \omega_{Y}\right)$ with a Hamiltonian $G$ action, an equivariant momentum map $F_{Y}$ and an embedding $i: G \cdot x \hookrightarrow Y$ such that $i^{*} \omega_{Y}=\left.\omega_{M}\right|_{G \cdot x}$, the symplectic normal bundle of $i$ is the same as that of $G \cdot x \hookrightarrow M$ and $F_{Y}(x)=\alpha$. The constant rank embedding theorem, Theorem 9, would then guarantee that there are neighborhoods $U$ of $G \cdot x$ in $M, U_{Y}$ of $G \cdot x$ in $Y$ and a $G$ equivariant diffeomorphism $\phi: U_{Y} \rightarrow U$ such that $\phi^{*} \omega_{M}=\omega_{Y}$ and $\phi^{*} F=F_{Y}$.

Proposition 11. Let $F: M \rightarrow \mathrm{g}^{*}$ be a momentum map for a Hamiltonian action of a Lie group $G$ on a symplectic manifold $\left(M, \omega_{M}\right)$. Then for any point $x \in M$ the restriction of the ambient symplectic form $\omega_{M}$ to the orbit $G \cdot x$ equals the pullback by the momentum map $F$ of the symplectic form on the coadjoint orbit through $F(x)$ :

$$
\left.\omega_{M}\right|_{G \cdot x}=\left.F^{*} \omega_{G \cdot F(x)}\right|_{G \cdot x}
$$

where $\omega_{G \cdot F(x)}$ is the Kirillov-Kostant-Souriau $(K K S)$ symplectic form on the coadjoint orbit of $F(x)$ in $\mathrm{g}^{*}$.

Proof. Let $\alpha=F(x)$. For a vector $\xi \in \mathrm{g}$ let $\xi_{M}$ denote the corresponding vector field on $M$ induced by the action of $G$ and $\xi_{\mathrm{g}^{*}}$ the corresponding 
vector field on $\mathrm{g}^{*}$ induced by the coadjoint action. By the definition of the momentum map, we have for any $\xi, \eta \in \mathrm{g}$

$$
\omega_{M}(x)\left(\xi_{M}(x), \eta_{M}(x)\right)=\left\langle\xi, d F_{x}\left(\eta_{M}(x)\right)\right\rangle .
$$

By the equivariance of $F$, we have $d F_{x}\left(\eta_{M}(x)\right)=\eta_{\mathrm{g}^{*}}(F(x))=\eta_{\mathrm{g}^{*}}(\alpha)$. Finally,

$$
\left\langle\xi, \eta_{\mathrm{g}^{*}}(\alpha)\right\rangle=\langle(-a d \eta) \xi, \alpha\rangle=\langle[\xi, \eta], \alpha\rangle=\omega_{G \cdot \alpha}\left(\xi_{\mathrm{g}^{*}}(\alpha), \eta_{\mathrm{g}^{*}}(\alpha)\right)
$$

and we are done.

Remark. The proposition allows us to take a more uniform view of regular reduction and, in particular, to make sense of the shifting trick. Suppose a momentum map $F: M \rightarrow \mathrm{g}^{*}$ is transversal to a locally closed orbit $G \cdot \alpha \subset \mathrm{g}^{*}$ and that the action of $G$ on the preimage $F^{-1}(G \cdot \alpha)$ is free. Then by the proposition the form $\left.\left(\omega-F^{*} \omega_{G \cdot \alpha}\right)\right|_{F^{-1}(G \cdot \alpha)}$ is basic and descends to a symplectic form on the quotient. This form on the quotient is the Marsden-Weinstein-Meyer reduced form.

Corollary 12. The symplectic perpendicular $T_{x}(G \cdot x)^{\omega_{M}}$ to the tangent space at $x$ of the orbit through $x$ intersects the tangent space in the $G_{\alpha}$ directions:

$$
T_{x}(G \cdot x)^{\omega_{M}} \cap T_{x}(G \cdot x)=T_{x}\left(G_{\alpha} \cdot x\right) .
$$

Here $G_{\alpha}$ is the isotropy group of $\alpha=F(x)$, the image of $x$ under the momentum map $F$.

Corollary 12 says that the subspace $\left(\mathrm{g}_{\alpha}\right)_{M}(x):=\left\{\xi_{M}(x): \xi \in \mathrm{g}_{\alpha}=\right.$ $\left.\operatorname{Lie}\left(G_{\alpha}\right)\right\}$ is the null space of the form $\left.\omega_{M}(x)\right|_{T_{x}(G \cdot x)}$. Note that since $F$ is equivariant, the isotropy group $G_{x}$ of $x$ is contained in $G_{\alpha}$.

Choose a $G_{x}$ invariant splitting

$$
\mathrm{g}=\mathrm{g}_{\alpha} \oplus \mathrm{s}
$$

(we use the fact that $G_{x}$ is compact). Then $\left.\omega_{M}(x)\right|_{s_{M}(x)}$ is nondegenerate. So $\mathrm{s}_{M}(x)$ is a symplectic subspace of $\left(T_{x} M, \omega_{M}(x)\right)$ isomorphic to the tangent space of the coadjoint orbit through $F(x)$. (Note that $\left.\omega_{M}(x)\right|_{\mathrm{s}_{M}(x)} \simeq$ $\omega_{G \cdot \alpha}(\alpha), \alpha=F(x)$.) Also the symplectic perpendicular $T_{x}(G \cdot x)^{\omega}$ contains $\left(\mathrm{g}_{\alpha}\right)_{M}(x)$. Pick a $G_{x}$ invariant splitting $T_{x}(G \cdot x)^{\omega}=\left(\mathrm{g}_{\alpha}\right)_{M}(x) \oplus V$. Then $V$ is a symplectic subspace in $\left(T_{x} M, \omega_{M}(x)\right)$. Note that $V$ is isomorphic to the quotient $T_{x}(G \cdot x)^{\omega} /\left(T_{x}(G \cdot x)^{\omega} \cap T_{x}(G \cdot x)\right)$, which is a typical fiber of the symplectic normal bundle of the orbit $G \cdot x$ in $M$. Let $\omega_{V}=\left.\omega_{M}(x)\right|_{V}$.

Since $V$ and $\mathrm{s}_{M}(x)$ are both symplectic and $G_{x}$ invariant, the symplectic perpendicular $\left(V \oplus \mathrm{s}_{M}(x)\right)^{\omega}$ is also symplectic and $G_{x}$ invariant. The 
symplectic perpendicular contains $\left(\mathrm{g}_{\alpha}\right)_{M}(x)$, which is null in it. Hence, by dimension count, this space is Lagrangian in the symplectic perpendicular. We conclude that $\left(T_{x} M, \omega_{M}(x)\right)$ splits as a direct sum of three symplectic subspaces:

$$
\left(T_{x} M, \omega_{M}(x)\right)=\left(V, \omega_{V}\right) \oplus\left(\mathrm{s}_{M}(x), \omega_{G \cdot \alpha}(\alpha)\right) \oplus\left(\left(\mathrm{g}_{\alpha}\right)_{M}(x) \oplus\left(\mathrm{g}_{\alpha}\right)(x)^{*}\right),
$$

and the splitting is $G_{x}$ invariant. The symplectic form on the last summand is the canonical form on the product of a vector space with its dual.

The tangent space at $x$ to the total space $Y$ of the associated bundle $\pi: G \times_{G_{x}}\left(\left(\mathrm{~g}_{\alpha} / \mathrm{g}_{x}\right)^{*} \oplus V\right) \rightarrow G \cdot x$ (we think of the orbit $G \cdot x$ as being embedding in the bundle as the zero section) is

$T_{x} Y \simeq T_{x}(G \cdot x) \oplus\left(\mathrm{g}_{\alpha} / \mathrm{g}_{x}\right)^{*} \oplus V \simeq\left(\mathrm{s} / \mathrm{g}_{x}\right) \oplus\left(\mathrm{g}_{\alpha} / \mathrm{g}_{x}\right) \oplus\left(\mathrm{g}_{\alpha} / \mathrm{g}_{x}\right)^{*} \oplus V \simeq T_{x} M$.

We now constract a closed $G$ invariant two form $\tau$ on the total space $Y$ of the associated bundle such that

$$
\left(T_{x}\left(G \times_{G_{x}}\left[\left(\mathrm{~g}_{\alpha} / \mathrm{g}_{x}\right)^{*} \oplus V\right]\right), \tau(x)\right)=\left(T_{x} M, \omega(x)\right) .
$$

The form $\tau$ is going to be the sum of three terms. We construct the first term $\tau_{1}$ by first pulling back by the momentum map $F$ the KKS symplectic form $\omega_{G \cdot \alpha}$ on the coadjoint orbit through $\alpha=F(x)$. We then pull it back by the bundle projection map $\pi: Y \rightarrow G \cdot x$, so $\tau_{1}=\pi^{*}\left(\left.F\right|_{G \cdot x}\right)^{*} \omega_{G \cdot \alpha}$. At the point $x$ the form $\tau_{1}$ is a nondegenerate two form on the subspace $\mathrm{s} / \mathrm{g}_{x} \simeq \mathrm{s}_{M}(x)$.

To construct the second and the third terms observe that the diagram

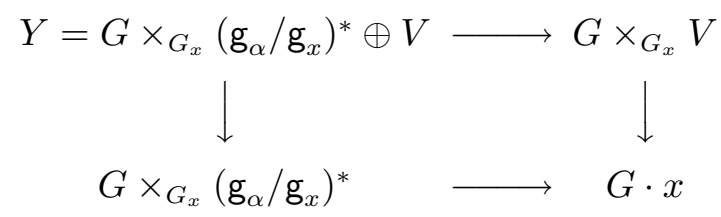

commutes. So we can think of $Y$ as a vector bundle over $G \times_{G_{x}} V$ with typical fiber $\left(\mathrm{g}_{\alpha} / \mathrm{g}_{x}\right)^{*}$ or as a vector bundle over $G \times{ }_{G \cdot x}\left(\mathrm{~g}_{\alpha} / \mathrm{g}_{x}\right)^{*}$ with typical fiber $V$. Therefore a form on the total space of $G \times{ }_{G_{x}} V$ or of $G \times{ }_{G \cdot x}\left(\mathrm{~g}_{\alpha} / \mathrm{g}_{x}\right)^{*}$ may be thought of as a form on the manifold $Y$.

To construct the second term $\tau_{2}$ we embed $G \times_{G_{x}}\left(\mathrm{~g}_{\alpha} / \mathrm{g}_{x}\right)^{*}$ into the cotangent bundle of the orbit $G \cdot x$. The $G_{x}$ equivariant splitting $\mathrm{g}=\mathrm{s} \oplus \mathrm{g}_{\alpha}$ chosen above gives rise to a $G_{x}$ equivariant projection

$$
\mathrm{g} \rightarrow \mathrm{g}_{\alpha}
$$

which induces an embedding

$$
j:\left(\mathrm{g}_{\alpha} / \mathrm{g}_{x}\right)^{*} \hookrightarrow\left(\mathrm{g} / \mathrm{g}_{x}\right)^{*}
$$


and thereby an embedding $j$ of the associated vector bundles

$$
j: G \times_{G_{x}}\left(\mathrm{~g}_{\alpha} / \mathrm{g}_{x}\right)^{*} \rightarrow G \times_{G_{x}}\left(\mathrm{~g} / \mathrm{g}_{x}\right)^{*} \simeq T^{*}(G \cdot x) .
$$

The pull-back by $j$ of the canonical symplectic form $\omega_{T^{*}(G \cdot x)}$ on the cotangent bundle of the orbit $G \cdot x$ is a closed two form on $G \times_{G_{x}}\left(\mathrm{~g}_{\alpha} / \mathrm{g}_{x}\right)^{*}$, hence it gives rise to a closed two form $\tau_{2}$ on $Y$.

The construction of the third term $\tau_{3}$ is an example of minimal coupling of Sternberg [GS2]. We first refine the splitting $\mathrm{g}=\mathrm{s} \oplus \mathrm{g}_{\alpha}$ to a $G_{x}$ equivariant splitting

$$
\mathrm{g}=\mathrm{g}_{x} \oplus \mathrm{m} \oplus \mathrm{s}
$$

(with $\mathrm{g}_{\alpha}=\mathrm{g}_{x} \oplus \mathrm{m}$ ). Let $A_{0}: \mathrm{g} \rightarrow \mathrm{g}_{x}$ be the corresponding $G_{x}$ equivariant projection. It defines a left $G$ invariant $\mathrm{g}_{x}$-valued one form $A$ on $G$. The form $A$ is a connection one form for the principal $G_{x}$ bundle $G \rightarrow G \cdot x$. Let $F_{V}: V \rightarrow \mathrm{g}_{x}^{*}$ denote the momentum map arising from the linear symplectic action of $G_{x}$ on $\left(V, \omega_{V}\right)$. Consider the following two form on $G \times V$ :

$$
\tilde{\tau}_{3}=d\left\langle A, F_{V}\right\rangle+\omega_{V}
$$

The form is $G_{x}$ invariant. It is not hard to check that it is basic for the projection $G \times V \rightarrow G \times \times_{G_{x}} V$. Denote the corresponding closed two form on $G \times_{G_{x}} V$ and hence on $Y$ by $\tau_{3}$. Note that the value of $\tau_{3}$ at $x$ on $V \subset$ $T_{x} Y=T_{x} M$ is the form $\omega_{V}$ and the restriction of $\tau_{3}(x)$ to the other two summand is zero. (This is because $F_{V}$ is a homogeneous quadratic map on $V$, so $F_{V}(0)=0$ and $d F_{V}(0)=0$. Consequently at a point $(g, 0) \in G \times V$ we have $d\left\langle A, F_{V}\right\rangle(g, 0)=\left\langle d A, F_{V}\right\rangle(g, 0)+\left\langle A \wedge d F_{V}\right\rangle(g, 0)=0+0=0$. $)$

We conclude that $\left(\tau_{1}+\tau_{2}+\tau_{3}\right)(x)=\omega(x)$. Let $\tau$ be the sum $\tau_{1}+\tau_{2}+\tau_{3}$. By construction $\tau$ is a closed $G$ invariant two form on the total space of $Y=G \times_{G_{x}}\left(\left(\mathrm{~g}_{\alpha} / \mathrm{g}_{x}\right)^{*} \oplus V\right)$ which is non-degenerate at the points of the zero section $G \cdot x$. Thus $\tau$ is non-degenerate in some ( $G$ invariant) neighborhood $Y_{0}$ of the zero section. Note that by construction we have $\left.\tau\right|_{G \cdot \alpha}=F^{*} \omega_{G \cdot x}=$ $\left.\omega\right|_{G \cdot x}$ and the symplectic normal bundle of the embedding $G \cdot x \hookrightarrow\left(Y_{0}, \tau\right)$ is $G \times{ }_{G_{x}} V$ which is the symplectic normal bundle of the embeddding $G \cdot x \hookrightarrow$ $(M, \omega)$. The constant rank embedding theorem says that if we shrink the neighborhood $Y_{0}$ a bit more, we will have a $G$ equivariant map $\psi: Y_{0} \rightarrow M$ which is a diffeomorphism onto its image and has the properties that $\left.\psi\right|_{G \cdot x}$ is the identity map and $\psi^{*} \omega=\tau$. That is to say, a neighborhood of the zero section $G \cdot x$ in the associated bundle $\left(G \times_{G_{x}}\left[\left(\mathrm{~g}_{\alpha} / \mathrm{g}_{x}\right)^{*} \oplus V\right], \tau\right)$ is the promised "Darboux coordinate patch adapted to the action of the Lie group G."

Our next step is compute a momentum map $F_{Y}$ for the action of $G$ on $(Y, \tau)$. The requirement that $F_{Y}(x)=F(x)=\alpha$ would then ensure that 
$F_{Y}=F \circ \psi$, where $F: M \rightarrow \mathrm{g}^{*}$ is the original momentum map. This would finally allow us to get on with computing the reduced space $F^{-1}(G \cdot \alpha) / G$.

A momentum map is traditionally defined for actions that preserve nondegenerate two forms. One can extend this definition to actions that preserve arbitrary two forms as long as the contractions of the vector fields induced by the action with the form are exact. For example, consider the form $\tau_{1}=\pi^{*}\left(\left.F\right|_{G \cdot x}\right)^{*} \omega_{G \cdot \alpha}$ on the manifold $Y$, where $\pi: Y \rightarrow G \cdot x$ is the vector bundle projection. For any $\xi \in \mathrm{g}$ we have

$$
\left.\xi_{Y}\right\lrcorner \tau_{1}=d\left\langle\xi, \pi^{*}\left(\left.F\right|_{G \cdot x}\right)\right\rangle .
$$

Hence $F_{1}=\pi^{*}\left(\left.F\right|_{G \cdot x}\right)$ is a momentum map for the action of $G$ on $\left(Y, \tau_{1}\right)$. Note that $F_{1}([g, \lambda, v])=g \cdot \alpha$ where $[g, \alpha, v]$ is the class of $(g, \lambda, v) \in G \times$ $\left(\mathrm{g}_{\alpha} / \mathrm{g}_{x}\right)^{*} \times V$ in the associated bundle $Y$.

Similarly since the lifted action of $G$ on the cotangent bundle $T^{*}(G \cdot x)=$ $G \times{ }_{G_{x}} \mathrm{~g}_{x}^{\circ}\left(\mathrm{g}_{x}^{\circ}\right.$ is the annihilator of $\mathrm{g}_{x}$ in $\left.\mathrm{g}^{*}\right)$ is Hamiltonian with momentum map sending the class $[g, \lambda]$ of $(g, \lambda) \in G \times \mathrm{g}_{x}^{\circ}$ to $g \cdot \lambda$, the map $F_{2}: G \times \times_{G_{x}}$ $\left[\left(\mathrm{g}_{\alpha} / \mathrm{g}_{x}\right)^{*} \oplus V\right] \rightarrow \mathrm{g}^{*}$ sending the class $[g, \lambda, v]$ to $g \cdot j(\lambda)$ is a momentum map for the action of $G$ on $\left(Y, \tau_{2}\right)$. Recall that $j:\left(\mathrm{g}_{\alpha} / \mathrm{g}_{x}\right)^{*} \rightarrow\left(\mathrm{g} / \mathrm{g}_{x}\right)^{*}$ is defined by the choice of the splitting $\mathrm{g}=\mathrm{g}_{\alpha} \oplus \mathrm{s}$ made earlier.

Let us also compute a momentum map for the action of $G$ on $\left(Y, \tau_{3}\right)$. Note first that the action of $G$ on $G \times V$ given by $g \cdot(a, v)$ preserves the form $\tilde{\tau}_{3}=d\left\langle A, F_{V}\right\rangle+\omega_{V}$. So for $\xi \in \mathrm{g}$ the induced vector field $\xi_{G \times V}=\xi_{G}$ is a right invariant vector field on $G$ and

$$
\left.\left.\left.0=\left(\xi_{G}\right\lrcorner d+d \xi_{G}\right\lrcorner\right)\left\langle A, F_{V}\right\rangle=\xi_{G}\right\lrcorner d\left\langle A, F_{V}\right\rangle+d\left\langle A\left(\xi_{G}\right), F_{V}\right\rangle .
$$

Since $\xi_{G}$ is right invariant and $A$ is left invariant, $A\left(\xi_{G}\right)(g)=A_{0}\left(A d\left(g^{-1}\right) \xi\right)$. It follows that a momentum map for the action of $G$ on $\left(G \times V, \tilde{\tau}_{3}\right)$ is given by $(g, v) \mapsto g \cdot i\left(F_{V}(v)\right)$ where $i: \mathrm{g}_{x}^{*} \rightarrow \mathrm{g}^{*}$ is the transpose of the projection $\mathrm{g} \rightarrow \mathrm{g}_{x}$. Therefore $F_{3}:\left(Y, \tau_{3}\right) \rightarrow \mathrm{g}^{*}$, a momentum map for the action of $G$ on $\left(Y, \tau_{3}\right)$, is given by

$$
F_{3}([g, \lambda, v])=g \cdot i\left(F_{V}(v)\right) .
$$

The upshot of these computations is that $F_{Y}=F_{1}+F_{2}+F_{3}$ is a momentum map for the action of $G$ on $\left(Y, \tau=\tau_{1}+\tau_{2}+\tau_{3}\right)$, that is to say

$$
F_{Y}([g, \lambda, v])=g \cdot\left(\alpha+j(\lambda)+i\left(F_{V}(v)\right)\right)
$$

where $i: \mathrm{g}_{x}^{*} \hookrightarrow \mathrm{g}^{*}$ and $j:\left(\mathrm{g}_{\alpha} / \mathrm{g}_{x}\right)^{*} \hookrightarrow \mathrm{g}_{x}^{\circ}$ are induced by an $G_{x}$ equivariant splitting

$$
\mathrm{g}=\mathrm{g}_{\alpha} \oplus \mathrm{s}=\mathrm{g}_{x} \oplus \mathrm{m} \oplus \mathrm{s}
$$


The proposition below is a key computation.

Proposition 13. Assume that the coadjoint orbit through $\alpha=F(x)$ is locally closed. Then for a small enough neighborhood $Y_{0}$ of the orbit $G \cdot x$ in the model space $Y$, the intersection of the set $F_{Y}^{-1}(G \cdot \alpha)$ with the neighborhood $Y_{0}$ is of the form

$$
F_{Y}^{-1}(G \cdot \alpha) \cap Y_{0}=\left\{[g, \lambda, v] \in Y_{0}: \lambda=0 \quad \text { and } \quad F_{V}(v)=0\right\} .
$$

Proof. We write

$$
F_{Y}: G \times_{G_{x}}\left(\left(\mathrm{~g}_{\alpha} / \mathrm{g}_{x}\right)^{*} \times V\right) \rightarrow \mathrm{g}^{*}, \quad[g, \lambda, v] \mapsto g \cdot\left(\alpha+i(\lambda)+i\left(F_{V}(v)\right)\right)
$$

as a composition of two maps:

$$
b: G \times_{G_{x}}\left(\left(\mathrm{~g}_{\alpha} / \mathrm{g}_{x}\right)^{*} \times V\right) \rightarrow G \times_{G_{x}} \mathrm{~g}_{\alpha}^{*}, \quad[g, \lambda, v] \mapsto\left[g, \lambda+i_{\alpha}\left(F_{V}(v)\right)\right]
$$

and

$$
\mathcal{E}: G \times_{G_{x}} \mathrm{~g}_{\alpha}^{*} \rightarrow \mathrm{g}^{*}, \quad[g, \nu] \mapsto g \cdot(\alpha+k(\nu)) .
$$

Here $i_{\alpha}: \mathrm{g}_{x}^{*} \hookrightarrow \mathrm{g}_{\alpha}^{*}$ is the $G_{x}$ equivariant embedding defined by the $G_{x}$ equivariant splitting $\mathrm{g}_{\alpha}=\mathrm{g}_{x} \oplus \mathrm{m}$ chosen earlier, $\left(\mathrm{g}_{\alpha} / \mathrm{g}_{x}\right)^{*}$ is identified with the annihilator of $\mathrm{g}_{x}$ in $\mathrm{g}_{\alpha}^{*}$ and $k: \mathrm{g}_{\alpha}^{*} \hookrightarrow \mathrm{g}^{*}$ is the $G_{x}$ equivariant embedding corresponding to the splitting $\mathrm{g}=\mathrm{g}_{\alpha} \oplus \mathrm{s}$. At the points of the form $[g, 0]$ the map $\mathcal{E}$ is a submersion. By assumption the coadjoint orbit $G \cdot \alpha$ is embedded. Therefore the preimage $\mathcal{E}^{-1}(G \cdot \alpha)$ is an embedded submanifold of $G \times_{G_{x}} \mathrm{~g}_{\alpha}^{*}$ of codimension $\operatorname{dim} g_{\alpha}$. It follows that the zero section of $G \times{ }_{G_{x}} \mathrm{~g}_{\alpha}^{*}$ is a collection of connected components of the preimage of the orbit. Since the preimage is embedded, there is a neighborhood $\mathcal{U}$ of the zero section such that $\mathcal{E}^{-1}(G \cdot \alpha) \cap \mathcal{U}$ is the zero section. Let $Y_{0}=b^{-1}(\mathcal{U})$. Then

$$
F_{Y}^{-1}(G \cdot \alpha) \cap Y_{0}=b^{-1}\left(\mathcal{U} \cap \mathcal{E}^{-1}(G \cdot \alpha)\right)=Y_{0} \cap b^{-1} \text { (zero section). }
$$

Clearly, $b^{-1}$ (zero section) $=\left\{[g, 0, v]: F_{V}(v)=0\right\}$ and we are done.

Corollary 14. For any subgroup $H<G$ and any $\alpha \in \mathrm{g}^{*}$ with the orbit $G \cdot \alpha$ locally closed, the set $F^{-1}(G \cdot \alpha) \cap M_{(H)}$ is a submanifold of $M$ of constant rank, the quotient $\left(M_{\alpha}\right)_{(H)}:=\left(F^{-1}(G \cdot \alpha) \cap M_{(H)}\right) / G$ is a symplectic manifold and the inclusion $\left(M_{\alpha}\right)_{(H)} \hookrightarrow M_{\alpha}:=F^{-1}(G \cdot \alpha) / G$ is a Poisson map.

Proof. Let $x$ be a point in the intersection $F^{-1}(G \cdot \alpha) \cap M_{(H)}$. It is no loss of generality to assume that the isotropy group $G_{x}$ of $x$ is $H$. Recall that there exists a $G$ invariant neighborhood $U$ of the orbit $G \cdot x$ in $M$, a $G$ invariant neighborhood $Y_{0}$ of the zero section in $Y=G \times \times_{G_{x}}\left(\left(\mathrm{~g}_{\alpha} / \mathrm{g}_{x}\right)^{*} \times V\right)$ and a $G$ 
equivariant diffeomorphism $\psi: Y_{0} \rightarrow U$ such that $\psi^{*} \omega=\left.\tau\right|_{Y_{0}}$ where $\tau$ is the closed two form on $Y$ constructed earlier. It follows that $\psi$ descends to a homeomorphism $\psi_{\alpha}:\left(Y_{0}\right)_{\alpha} \rightarrow U_{\alpha}$. Here $\left(Y_{0}\right)_{\alpha}:=\left(F_{Y}^{-1}(G \cdot \alpha) \cap Y_{0}\right) / G$ and $U_{\alpha}:=\left(U \cap F^{-1}(G \cdot \alpha)\right) / G$ are the reduced spaces. Moreover by construction the pull-back map $\psi_{\alpha}^{*}: C^{\infty}\left(\left(Y_{0}\right)_{\alpha}\right) \rightarrow C^{\infty}\left(U_{\alpha}\right)$ is an isomorphism of Poisson algebras, where $C^{\infty}\left(U_{\alpha}\right):=\left.C^{\infty}(U)^{G}\right|_{F^{-1}(G \cdot \alpha)}$ etc. Therefore it is enough to prove the statements of the corollary for the action of $G$ on $\left(Y_{0}, \tau\right)$.

It is convenient to ignore the distinction between the total space $Y$ and the neighborhood $Y_{0}$ of the zero section in $Y$. We note for future reference that the embedding of the symplectic slice $V$ into the model space $Y$ given by $v \mapsto[e, 0, v]$, where $e$ is the identity element of $G$, is symplectic, i.e., $\left.\tau\right|_{V}=\omega_{V}$. It is not hard to see that

$$
Y_{(H)}=G \times_{H}\left[\left(\mathrm{~g}_{\alpha} / \mathrm{h}\right)^{*} \times V\right]^{H}
$$

where $\left[\left(\mathrm{g}_{\alpha} / \mathrm{h}\right)^{*} \times V\right]^{H}$ denotes the subspace of $H$ fixed vectors (remember that $\left.G_{x}=H\right)$. It follows that $F_{Y}^{-1}(G \cdot \alpha) \cap Y_{(H)}=G \times_{H} V^{H} \simeq G / H \times V^{H}$. Since $\left.\tau\right|_{G / H \times V^{H}}=\left.\omega\right|_{G \cdot x}+\left.\omega_{V}\right|_{V^{H}}$ and $V^{H}$ is a symplectic subspace of $V$, we conclude that $F_{Y}^{-1}(G \cdot \alpha) \cap Y_{(H)}$ is a submanifold of $(Y, \tau)$ of constant rank and that the quotient $\left(F_{Y}^{-1}(G \cdot \alpha) \cap Y_{(H)}\right) / G$ is diffeomorphic to $V^{H}$.

Therefore $F^{-1}(G \cdot \alpha) \cap M_{(H)}$ is a submanifold of $(M, \omega)$ of constant rank and the quotient $\left(F^{-1}(G \cdot \alpha) \cap M_{(H)}\right) / G$ is a manifold. We also see that the form $\left.\left(\omega-F^{*} \omega_{G \cdot \alpha}\right)\right|_{F^{-1}(G \cdot \alpha) \cap M_{(H)}}$ is basic and descends to a form on the base locally isomorphic to $\left.\omega_{V}\right|_{V^{H}}$. Thus $\left(M_{\alpha}\right)_{(H)}$ is a symplectic manifold.

Finally observe that if a function $f$ on $Y$ is $G$ invariant then its restriction to $F_{Y}^{-1}(G \cdot \alpha) \cap Y_{(H)}=G / H \times V^{H}$ is completely determined by its restriction to $V^{H}$. It follows that the map $\left(M_{\alpha}\right)_{(H)} \hookrightarrow M_{\alpha}$ is Poisson.

To finish the proof of Theorem 7 we need to show that the decomposition of the reduced space $M_{G \cdot \alpha}=M_{\alpha}$ into symplectic pieces is locally finite and that the Hamiltonian flows of smooth function on the reduced space preserve the decomposition. The local finiteness of the decomposition of the reduced space follows from the local finiteness of the decomposition of the original manifold $M$ into orbit types which in turn follows from the existence of slices for proper group actions. The fact that the Hamiltonian flows preserve the symplectic pieces of the reduced space is a consequence of the fact that Hamiltonian flows of $G$ invariant functions on $M$ are $G$ equivariant and hence preserve the orbit types. This concludes the proof.

We now refine Theorem 7 in two different ways. The first refinement is a theorem that describes more precisely how the symplectic pieces fit together. The second one is a theorem that shows that the symplectic pieces can also 
be obtained by regular reduction, thus providing a way to reconstruct the reduced dynamics.

Theorem 15. Let $G$ be a Lie group acting properly and in a Hamiltonian fashion on a symplectic manifold $(M, \omega)$ with a corresponding equivariant momentum map $F: M \rightarrow \mathrm{g}^{*}$. Let $x$ be a point in the manifold $M$ and $\alpha=F(x)$. Assume that the coadjoint orbit $G \cdot \alpha$ is locally closed. Then locally the reduced space $M_{\alpha}=F^{-1}(G \cdot \alpha) / G$ is isomorphic to a neighborhood of the image of the origin in the space obtained by reduction at 0 of the symplectic slice $V:=T_{x}(G \cdot x)^{\omega} /\left(T_{x}(G \cdot x)^{\omega} \cap T_{x}(G \cdot x)\right)$ through the point $x$ with respect to the isotropy group $G_{x}$ of $x$.

Proof. As before it enough to prove the theorem at the point $x$ for the model space $(Y, \tau)$, where $Y=G \times_{G_{x}}\left(\left(\mathrm{~g}_{\alpha} / \mathrm{g}_{x}\right)^{*} \times V\right)$. Of course, as was mentioned previously, the form $\tau$ is only nondegenerate on some neighborhood of the zero section $G \cdot x$, so the Poisson bracket is only defined in that neighborhood. It would be a notational nightmare to keep track of the neighborhood so we will again pretend that $\tau$ is nondegenerate everywhere on $Y$.

Recall that the embedding of the symplectic slice $V$ into the model space $Y$ given by $v \mapsto[e, 0, v]$, where $e$ is the identity element of $G$, is symplectic, i.e., $\left.\tau\right|_{V}=\omega_{V}$. We now prove that the restriction $C^{\infty}(Y) \rightarrow C^{\infty}(V)$ induces an isomorphism of Poisson algebras $C^{\infty}\left(Y_{\alpha}\right) \rightarrow C^{\infty}\left(V_{0}\right)$ where $C^{\infty}\left(Y_{\alpha}\right):=$ $\left.C^{\infty}(Y)^{G}\right|_{F_{Y}^{-1}(G \cdot \alpha)}$ and $C^{\infty}\left(V_{0}\right):=\left.C^{\infty}(V)^{G_{x}}\right|_{F_{V}^{-1}(0)}$ are the algebras of smooth functions on the corresponding reduced spaces. It is easy to see that restriction to $V$ defines a surjective map $C^{\infty}(Y)^{G} \rightarrow C^{\infty}(V)^{G_{x}},\left.f \mapsto f\right|_{V}$. Indeed, any $G$ invariant function on $G \times{ }_{G_{x}}\left(\left(\mathrm{~g}_{\alpha} / \mathrm{g}_{x}\right)^{*} \times V\right)$ restricts to an $G_{x}$ invariant function on $\left(\mathrm{g}_{\alpha} / \mathrm{g}_{x}\right)^{*} \times V$ hence to a $G_{x}$ invariant function on $V$. Conversely, any $G_{x}$ invariant function on $V$ extends trivially to a $G \times G_{x}$ invariant function on $G \times\left(\mathrm{g}_{\alpha} / \mathrm{g}_{x}\right)^{*} \times V$, so the restriction map $C^{\infty}(Y)^{G} \rightarrow C^{\infty}(V)^{G_{x}}$ is surjective. In fact the same argument shows that the map $C^{\infty}\left(G \times_{G_{x}}(\{0\} \times V)\right)^{G} \rightarrow C^{\infty}(V)^{G_{x}}$ is bijective. Since $F_{Y}^{-1}(G \cdot \alpha) \cap V=$ $F_{V}^{-1}(0)$, it follows that the map

$$
\left.\left.C^{\infty}(Y)^{G}\right|_{F_{Y}^{-1}(G \cdot \alpha)} \rightarrow C^{\infty}(V)^{G_{x}}\right|_{F_{V}^{-1}(0)}
$$

induced by restriction to $V$ is bijective as well. The bijection is a Poisson map because $\left.\tau\right|_{V}=\omega_{V}$.

Theorem 15 shows that the decomposition of a reduced space into symplectic pieces is well behaved. The reason for this good behavior is that the decomposition of a vector space reduced at zero with respect to a linear action of a compact group forms a Whitney regular stratification. We now present the details. 
We recall the discussion in $[\mathbf{S L}]$. Let $\left(V, \omega_{V}\right)$ be a symplectic vector space, $K$ a compact Lie group and $K \rightarrow S p\left(V, \omega_{V}\right)$ a symplectic representation of $K$. As was mentioned earlier the $K$ momentum map $F_{V}: V \rightarrow \mathrm{k}^{*}$ that sends zero to zero is a quadratic polynomial. The reduced space at zero $V_{0}=F_{V}^{-1}(0) / K$ can be described as a semi-algebraic set. To this end consider the algebra $\mathbf{R}[V]^{K}$ of $K$ invariant polynomials on $V$. It is well known that the algebra is finitely generated. A deep result due to G. Schwarz [Sch] asserts that the algebra of invariant functions $C^{\infty}(V)^{K}$ is also finitely generated in the following sense. Let $p_{1}, \ldots, p_{n}$ be a minimal set of generators of the algebra of invariant polynomials and let $p: V \rightarrow \mathbf{R}^{n}$ be given by $p(v)=\left(p_{1}(v), \ldots, p_{n}(v)\right)$. Schwarz's theorem asserts that the smooth invariant functions on $V$ are the compositions of smooth functions on $\mathbf{R}^{n}$ with the invariant map $p$, i.e.,

$$
C^{\infty}(V)^{K}=p^{*} C^{\infty}\left(\mathbf{R}^{n}\right) .
$$

Since $K$ invariant functions separate orbits the induced map $\bar{p}: V / K \rightarrow \mathbf{R}^{n}$ is injective. In fact it is a proper embedding (see for example $[\mathbf{B}]$ ) and the image $\bar{p}(V / K)=p(V)$ is, by the Tarski-Seidenberg theorem, a semi-algebraic subset of $\mathbf{R}^{n}$.

It is also easy to see that the map $\bar{p}$ embeds the reduced space $V_{0}$ as a semi-algebraic subset. Indeed, let $\|\cdot\|$ be a norm on the dual of the Lie algebra $\mathrm{k}^{*}$ defined by a $K$ invariant inner product. Then $\left\|F_{V}\right\|^{2}$ is an invariant polynomial on $V$. So there is a polynomial $f$ on $\mathbf{R}^{n}$ such that $\left\|F_{V}\right\|^{2}=f \circ p$. Since $\left(\left\|F_{V}\right\|^{2}\right)^{-1}(0)=F_{V}^{-1}(0)$ we have $\bar{p}\left(V_{0}\right)=\{f=0\} \cap \bar{p}(V)$. Thus $\bar{p}\left(V_{0}\right)$ is a semi-algebraic set. Note that in complete analogy with Schwarz's theorem the embedding map $\bar{p}: V_{0} \rightarrow \mathbf{R}^{n}$ induces a surjective map $\bar{p}^{*}: C^{\infty}\left(\mathbf{R}^{n}\right) \rightarrow C^{\infty}\left(V_{0}\right)$, where as before $C^{\infty}\left(V_{0}\right)$ denotes the algebra of smooth functions on the reduced space, $C^{\infty}\left(V_{0}\right)=\left.C^{\infty}(V)^{K}\right|_{F_{V}^{-1}(0)}$.

It was shown in $[\mathbf{S L}]$ that $\bar{p}$ embeds symplectic pieces of the reduced space $V_{0}$ into smooth submanifolds of $\mathbf{R}^{n}$. This defines a decomposition of the semialgebraic set $\bar{p}\left(V_{0}\right)$ into smooth manifolds. It was also shown (loc. cit.) that this decomposition of $\bar{p}\left(V_{0}\right)$ satisfies the Whitney regularity condition.

Thus Theorem 15 asserts that the decomposition of reduced spaces into symplectic pieces defined by orbit type is a stratification (in a technical sense) and that the stratification is locally Whitney regular. This excludes more pathological singular spaces such as a cone over the integers (not locally finite) or the set

$$
X=\left\{(x, y) \in \mathbf{R}^{2}: x=0,-1 \leq y \leq 1\right\} \cup\left\{(x, y) \in \mathbf{R}^{2}: x>0, y=\sin \frac{1}{x}\right\}
$$

which is connected but not path connected. 
The next theorem is useful in reconstructing the original dynamics from the dynamics in the reduced space.

Theorem 16. Let $G$ be a Lie group acting properly and in a Hamiltonian way on a symplectic manifold $(M, \omega)$ with a corresponding equivariant momentum amp $F: M \rightarrow \mathrm{g}^{*}$. Let $x$ be a point in the manifold $M, H$ the isotropy group of $x$ and $\alpha=F(x)$.

The manifold

$$
M_{H}:=\left\{m \in M: G_{m}=H\right\}
$$

is a symplectic submanifold of $M$. The normalizer $N$ of $H$ in $G$ preserves $M_{H}$ and the quotient group $L=N / H$ acts freely. This action of $L$ on $M_{H}$ is Hamiltonian and the reduced space $\left(M_{H}\right)_{\alpha_{0}}$, for an appropriate vector $\alpha_{0} \in \mathrm{I}^{*}$ is isomorphic to the symplectic piece $\left(M_{\alpha}\right)_{(H)}=\left(F^{-1}(G \cdot \alpha) \cap M_{(H)}\right) / G$ provided the orbit $G \cdot \alpha$ is locally closed.

Remark. Note that the action of $L$ on $M_{H}$ is free by construction, so the manifold $\left(M_{H}\right)_{\alpha_{0}}$ is obtained by regular Marsden-Weinstein-Meyer reduction.

Before a proof of Theorem 16 it would be useful to identify ${ }^{*}$, the dual of Lie algebra of the group $L$, with a subspace of $\mathrm{g}^{*}$. This is done in the following lemma. Note that we do not assume that the group $H$ is connected.

Lemma 17. Suppose $H$ is a compact subgroup of a Lie group $G$ and $N(H)$ is its normalizer in $G$. Then the dual of the Lie algebra of the group $L=$ $N(H) / H$ is naturally isomorphic to the subspace $\left(\mathrm{h}^{\circ}\right)^{H}$ of $H$-fixed vectors in the annihilator of $\mathrm{h}=\operatorname{Lie}(H)$ in $\mathrm{g}^{*}=\operatorname{Lie}(G)^{*}$, i.e.,

$$
\mathrm{I}^{*} \simeq\left(\mathrm{h}^{\circ}\right)^{H} .
$$

Proof. Note first that for any representation $V$ of the compact Lie group $H$, the dual $\left(V^{H}\right)^{*}$ of the subspace of $H$-fixed vectors is isomorphic to $\left(V^{*}\right)^{H}$, the space of vectors in the dual representation fixed by $H$. Moreover, the natural projection $V^{*} \rightarrow\left(V^{H}\right)^{*}$, the transpose of the inclusion $V^{H} \hookrightarrow V$, furnishes an isomorphism $\left(V^{*}\right)^{H} \rightarrow\left(V^{H}\right)^{*}$.

The group $L$, as a manifold, is isomorphic to the submanifold of $H$ fixed points in the homogeneous space $G / H$. Therefore the Lie algebra of $L$ is isomorphic, as a vector space, to the space of $H$-fixed vectors in the tangent space $T_{e H}(G / H)$ to the identity coset. The cotangent space $T_{e H}^{*}(G / H)$ is naturally isomorphic to $(\mathrm{g} / \mathrm{h})^{*} \simeq \mathrm{h}^{\circ}$. The natural projection $T_{e H}^{*}(G / H) \rightarrow T_{e H}^{*}(N(H) / H)$ identifies $\left(\mathrm{h}^{\circ}\right)^{H} \simeq\left(T_{e H}^{*}(G / H)\right)^{H}$ with $T_{e H}^{*}(N(H) / H) \simeq \mathrm{I}^{*}$. 
Proof of Theorem 16. We show that the action of $L$ on $\left(M_{H},\left.\omega\right|_{M_{H}}\right)$ is Hamiltonian. It is no loss of generality to assume that the manifold $M_{H}$ is connected.

Recall that $x$ is a point in $M_{H}$ and $\alpha$ is its image under the $G$ momentum map $F$. We claim that the image of $M_{H}$ under $F$ lies in the affine plane $\left(\mathrm{h}^{\circ}\right)^{H}+\alpha$. Indeed, since $M_{H}$ is pointwise fixed by $H$ and $F$ is equivariant, the image $F\left(M_{H}\right)$ is also pointwise fixed by $H$. Also, for any vector $\xi \in \mathrm{h}$, any point $y \in M_{H}$ and any tangent vector $v \in T_{y} M_{H}$ we have

$$
\left\langle\xi, d F_{y}(v)\right\rangle=\omega(y)\left(\xi_{M}(y), v\right)=0
$$

since $\xi_{M}(y)=0$ for all $y \in M_{H}$. Thus $d F_{y}\left(T_{y} M_{H}\right) \subset \mathrm{h}^{\circ}$ for all $y \in M_{H}$ and so $F\left(M_{H}\right) \subset \mathrm{h}^{\circ}+\alpha$ since $F(x)=\alpha$.

We conclude that the map $F_{L}:=\pi \circ\left(\left.F\right|_{M_{H}}\right)$ is a momentum map for the action of $L$ on $\left(M_{H},\left.\omega\right|_{M_{H}}\right)$. Here $\pi:\left(\mathrm{h}^{\circ}\right)^{H}+\alpha \rightarrow \mathrm{I}^{*}$ is the natural projection.

Since $H$ is closed in $G$, its normalizer $N$ is also closed in $G$, so the action of $N$ on $M_{H}$ (and hence of $L$ ) is proper. Therefore the reduced space $\left(M_{H}\right)_{\alpha_{0}}:=F_{L}^{-1}\left(L \cdot \alpha_{0}\right) / L$ is a symplectic manifold. As was mentioned before, Proposition 11 allows us the following description of the reduced symplectic structure on $\left(M_{H}\right)_{\alpha_{0}}$. The form $\omega_{M_{H}}:=\left.\omega\right|_{M_{H}}$ is not basic when restricted to the principal $L$ bundle $F_{L}^{-1}\left(L \cdot \alpha_{0}\right)$, but the difference $\left.\left(\omega_{M_{H}}-F_{L}^{*} \omega_{L \cdot \alpha_{0}}\right)\right|_{F_{L}^{-1}\left(L \cdot \alpha_{0}\right)}$ is basic and descends to the reduced symplectic form on $\left(M_{H}\right)_{\alpha_{0}}$ (here $\omega_{L \cdot \alpha_{0}}$ is the symplectic form on the coadjoint orbit $\left.L \cdot \alpha_{0} \subset \mathrm{I}^{*}\right)$.

We are now ready to prove the main claim of the theorem: that the manifold $\left(M_{\alpha}\right)_{(H)}:=\left(F^{-1}(G \cdot \alpha) \cap M_{(H)}\right) / G$ is symplectically diffeomorphic to $\left(M_{H}\right)_{\alpha_{0}}$. Note first that $F_{L}^{-1}\left(L \cdot \alpha_{0}\right)=F^{-1}(N \cdot \alpha) \cap M_{H}$. So to establish the diffeomorphism, it is enough to show that

$$
\left(F^{-1}(N \cdot \alpha) \cap M_{H}\right) / N \simeq\left(F^{-1}(G \cdot \alpha) \cap M_{(H)}\right) / G .
$$

We computed the right hand side locally in the proof of Corollary 14 . We now compute the left hand side locally using the same model $\left(Y_{0}, \tau\right)$. (As before we will ignore the distinction between the neighborhood $Y_{0}$ and the whole space $Y$.) We will see that locally the reduced space $\left(M_{H}\right)_{\alpha_{0}}$ is modeled by the vector space $\left(V^{H},\left.\omega_{V}\right|_{V^{H}}\right)$. This will prove the theorem.

The manifold $Y_{H}$ of points in $Y$ with isotropy group $H$ is equal to $N \times_{H}$ $\left[\left(\mathrm{g}_{\alpha} / \mathrm{h}\right)^{*} \times V\right]^{H}$. An argument similar to the proof of Proposition 13 (the factoring of the map $F_{Y}$ through two maps) shows that

$$
Y_{0} \cap F_{Y}^{-1}(N \cdot \alpha)=Y_{0} \cap\left(N \times_{H} F_{V}^{-1}(0)\right) .
$$


It follows that

$$
\left(Y_{0}\right)_{H} \cap F_{Y}^{-1}(N \cdot \alpha)=N \times_{H} V^{H} \simeq L \times V^{H} .
$$

Therefore, locally,

$$
\left(M_{H}\right)_{\alpha_{0}} \simeq\left(V^{H},\left.\omega_{V}\right|_{V^{H}}\right)
$$

and we are done.

Actions of compact Lie groups: Coadjoint directions don't matter.

The proofs of Theorem 7, 15 and 16 were based on Marle's constant rank embedding theortem [Ma2], [Ma1]. However for compact symmetry groups we can also use a local normal form theorem due to Guillemin and Sternberg [GS1]. This normal form is based on the idea of symplectic cross-sections. It allows us to restrict our attention to the smallest symplectic submanifold containing a given fiber of the momentum map. This reduces the number of the degrees of freedom and the dimension of the symmetry group. As a result, for compact groups we only need to deal with reduction at zero values of the momentum maps which is described in [SL].

The symplectic cross-section theorem can be stated as follows.

Theorem 18 ([GS2, Theorem 26.7]). Let $(M, \omega)$ be a Hamiltonian $G$ space with momentum map $F: M \rightarrow \mathrm{g}^{*}$. Suppose $S$ is a submanifold of $\mathrm{g}^{*}$ passing through a point $\alpha \in \mathrm{g}^{*}$ satisfying $T_{\alpha} S \oplus T_{\alpha} G \cdot \alpha=\mathrm{g}^{*}$ and suppose that $S$ is $G_{\alpha}$ invariant. Then for a small enough $G_{\alpha}$ invariant neighborhood $B$ of $\alpha$ in $S$ the preimage $F^{-1}(B)$ is a symplectic submanifold of $M$ and the action of $G_{\alpha}$ on $F^{-1}(B)$ is Hamiltonian with momentum map being the restriction of $F$ followed by the projection onto $T_{\alpha} S \simeq \mathrm{g}_{\alpha}^{*}$, the dual of the Lie algebra of the isotropy group of $\alpha$.

Remark. Theorem 18 above does not assume that the group $G$ is compact. The main assumption of the theorem is that the tangent space to the orbit at $\alpha$ has a $G_{\alpha}$ invariant complement in $\mathrm{g}^{*}$. Clearly this is true for any point $\alpha$ if the group $G$ is compact. If $G$ is a real simple group and $\alpha$ is a semi-simple element (under the identification of $\mathrm{g}^{*}$ with $\mathrm{g}$ ), then again the tangent space to the orbit at $\alpha$ has a $G_{\alpha}$ invariant complement. If $\alpha$ is nilpotent then no such splitting exists.

Remark. Guillemin and Sternberg call the submanifold $R=F^{-1}(B)$ a symplectic cross-section. It has the property that for $m \in F^{-1}(\alpha)$ the $G_{\alpha}$ orbit is isotropic in the cross-section. Also the cross-section is the smallest 
symplectic submanifold of $M$ containing the fiber $F^{-1}(\alpha)$. Thus if the $\alpha$ fiber is a manifold then it is coisotropic in the cross-section. Therefore the Marsden-Weinstein-Meyer reduction away from zero can be thought of as a coisotropic reduction, but in a smaller manifold and for a smaller group. (Compare this with the shifting trick that enlarges the manifold and keeps the group the same.)

Remark. If the manifold $S$ is chosen carefully then the open neighborhood $B$ of $\alpha$ in $S$ can be quite large. For example if $\alpha$ lies in the interior of a Weyl chamber we can choose $S$ to be corresponding Cartan subalgebra and $B$ to be all of the interior of the Weyl chamber. Proving this fact will take us too far afield and we refer the reader to [GLS] for details.

Now suppose we have a $G$-invariant Hamiltonian $h$ on the manifold $M$, and let $R$ be a symplectic cross-section through a point $x$ in $M$. Then $\Xi_{h}$, the Hamiltonian vector field of $h$, preserves $R$. To see this observe that $R$ is a union of fibers of the momentum map, and the flow of $\Xi_{h}$ preserves the fibers. This means that $\left(R,\left.h\right|_{R}\right)$ is a $G_{\alpha}$-invariant subsystem of the original system (here as before $\alpha=F(x)$ and $G_{\alpha}$ is the isotropy group of $\alpha$ ). This is a precise way to say that we have "factored out" the coadjoint orbit $G \cdot \alpha$ directions.

In general, pushing the cross-section $R$ by the action of the group $G$ yields an open submanifold isomorphic to the symplectic fiber bundle

$$
R \longrightarrow G \times_{G_{\alpha}} R \longrightarrow G \cdot \alpha .
$$

Therefore we may think of the open submanifold as being fibered by lower dimensional invariant Hamiltonian systems which are all isomorphic by $G$ invariance of the total system. For instance, this point of view allows us to conclude that the subsystem $\left(R,\left.h\right|_{R}\right)$ has a stable $G_{\alpha}$-relative equilibrium if and only if the full system has a stable $G$-relative equilibrium. In other words, the coadjoint orbit directions are irrelevant as far as the relative equilibria are concerned or any other $G$-invariant features of the motion.

Example. Consider a particle in three space moving under the influence of a central force. Factoring out the coadjoint orbit directions amounts to fixing a direction of angular momentum. For a fixed direction of angular momentum the motion lies in a two plane. Therefore we can decompose phase space, $T^{*} \mathbf{R}^{3}$, as a family of cotangent bundles of two-planes parametrized by a two-sphere plus the set of points of zero angular momentum.

\section{Appendix.}

The goal of this section is to provide the reader with a number of proofs that are well known to experts but don't seem to be readily available in 
the literature. We start with the existence of invariant almost complex structures adapted to a given symplectic form (fact 7 of our digression on proper actions).

Proposition 19 (Existence of invariant almost complex structures adapted to an invariant form). Let $G$ be a Lie group acting properly on a manifold $P$, and preserving a symplectic form $\tau$. Then there exists a $G$ invariant almost complex structure $J$ adapted to $\tau$, i.e., $\tau(J \cdot, J \cdot)=(\cdot, \cdot)$ and $\tau(\cdot, J \cdot)$ is a Riemannian metric.

Proof. Recall a proof of existence of a complex structure tamed by a symplectic form in the setting of vector spaces. Let $V$ be a vector space and $\tau$ a skew-symmetric nondegenerate bilinear form. Choose a positive definite metric $g$. We have two isomorphisms:

$$
\tau^{\#}: V \rightarrow V^{*}, \quad v \mapsto \tau(v, \cdot)
$$

and

$$
g^{\#}: V \rightarrow V^{*}, \quad v \mapsto g(v, \cdot)
$$

Let $A=\left(g^{\#}\right)^{-1} \circ \tau^{\#}$. Then for any $v, w \in V$ we have

$$
\begin{aligned}
g(A v, w) & =\left\langle g^{\#} A v, w\right\rangle=\left\langle\tau^{\#} v, w\right\rangle \\
& =\tau(v, w)=-\tau(w, v)=-g(A w, v)=-g(v, A w),
\end{aligned}
$$

i.e., $A=-A^{*}$ where the adjoint is taken relative to the metric $g$. Therefore $-A^{2}=A A^{*}$ is diagonalizable and all eigenvalues are positive. Let $P$ be the positive square root of $-A^{2}$. For example we can define $P$ by

$$
P=\frac{1}{2 \pi \sqrt{-1}} \int_{\gamma}\left(-A^{2}-z\right)^{-1} \sqrt{z} d z,
$$

where $\sqrt{z}$ is defined via the branch cut along the negative real axis and $\gamma$ is a contour containing the spectrum of $-A^{2}$. It follows that $P$ commutes with $A$ and that

$$
\left(A P^{-1}\right)^{2}=A^{2} P^{-2}=A^{2}\left(-A^{2}\right)=-1 .
$$

The map $J=A P^{-1}$ is the desired complex structure.

Note that the same argument works if we consider a symplectic vector bundle $(E \rightarrow X, \tau)$. We choose a Riemannian metric $g$ on $E$ and consider a vector bundle map $A=\left(g^{\#}\right)^{-1} \circ \tau^{\#}$. We define $P: E \rightarrow E$ by essentially 
the same formula. For $x \in X$ the map $P_{x}: E_{x} \rightarrow E_{x}$ on the fiber above $x$ is given by

$$
P_{x}=\frac{1}{2 \pi \sqrt{-1}} \int_{\gamma_{x}}\left(-A_{x}^{2}-z\right)^{-1} \sqrt{z} d z .
$$

Note that since the spectrum of $A_{x}$ varies with the base point $x$ and since we don't assume the base is compact, we have to let the contour $\gamma_{x}$ vary with $x$ as well to make sure that the spectrum of $-A_{x}^{2}$ lies inside $\gamma_{x}$. The map $P$ so defined is a smooth vector bundle map that commutes with $A$ and we set the complex structure $J$ to be $A P^{-1}$.

Note finally that if a group $G$ acts on the vector bundle $E$ in a way that preserves the form $\tau$ and that covers a proper action on the base, we can choose our metric $g$ to be $G$ invariant. Then, by construction, the corresponding complex structure $J$ on $E$ is $G$ invariant as well.

The next theorem that we prove is an equivariant version of the relative Darboux theorem (fact 9 of our digression on proper actions).

Theorem 6 (Relative Darboux). Let $X$ be a submanifold of a manifold $Y$. Let $\omega_{0}$ and $\omega_{1}$ be given symplectic forms on $Y$ such that $\omega_{0}(x)=\omega_{1}(x)$ for each $x \in X$. Then there exist neighborhoods $\mathcal{U}_{0}$ and $\mathcal{U}_{1}$ of $X$ and a diffeomorphism $\psi: \mathcal{U}_{0} \longrightarrow \mathcal{U}_{1}$ such that the pull back of $\omega_{1}$ by $\psi$ is $\omega_{0}$ and $\psi$ is the identity on $X$. If a Lie group $G$ acts properly on $Y$, preserves $X, \omega_{0}$ and $\omega_{1}$, then we can arrange that the neighborhoods $\mathcal{U}_{0}$ and $\mathcal{U}_{1}$ are $G$-invariant and that the diffeomorphism $\psi$ is G-equivariant.

Proof. First suppose that we can find a one form $\zeta$ on a tubular neighborhood of $X$ such that

1. $\omega_{1}-\omega_{0}=d \zeta$.

2. $\zeta$ vanishes identically on $X$.

3. $\zeta$ is $G$-invariant.

Since at the points of $X$, the form $\omega_{t}:=t \omega_{0}+(1-t) \omega_{1}$ is equal to $\omega_{0}$, it is nondegenerate at the points of $X$ for $0 \leq t \leq 1$. Therefore $\omega_{t}$ is nondegenerate for all $t \in[0,1]$ in neighborhood of $X$. On this neighborhood the equation

$$
\left.\xi_{t}\right\lrcorner \omega_{t}=\zeta
$$

defines a time dependent vector field $\xi_{t}$. The vector field is $G$-invariant and vanishes identically on $X$. The definition of the vector field $\xi_{t}$ is rigged in such a way as to ensure that its time $t$ flow $\varphi_{t}$ satisfies

$$
\frac{d}{d t} \varphi_{t}^{*} \omega_{t}=\omega_{0}
$$


Indeed, if (3) holds then, since $\dot{\omega}_{t}=\omega_{0}-\omega_{1}=-\zeta$ and since $d \omega_{t}=0$ we have $\left.\left.d\left(\xi_{t}\right\lrcorner d \omega_{t}\right)+\xi_{t}\right\lrcorner d \omega_{t}=-\dot{\omega}_{t}$, so

$$
\varphi_{t}^{*}\left(\mathcal{L}_{\xi_{t}} \omega_{t}+\dot{\omega}_{t}\right)=0
$$

which implies Equation (4). The time one map $\varphi=\varphi_{1}$ of the flow of $\xi_{t}$ is defined on some open neighborhood $\mathcal{W}$ of $X$ because it is defined on some open ball about each point of $X$. Therefore the flow is defined on the union $\mathcal{U}_{0}$ of $G$-translates of $\mathcal{W}$, that is, $\mathcal{U}_{0}=\cup_{g \in G} g \cdot \mathcal{W}$. Let $\mathcal{U}_{1}$ be the image of $\mathcal{U}_{0}$ under the time one map $\psi$. Then $\psi: \mathcal{U}_{0} \longrightarrow \mathcal{U}_{1}$ is the desired map.

It remains to prove the existence of the $G$-invariant one form $\zeta$ which vanishes on $X$ and satisfies $\omega_{1}-\omega_{0}=d \zeta$. Since $G$ acts properly, the isotropy subgroup of a point $x$ in $X$ is compact. Moreover, $G$ acts by vector bundle maps on the normal bundle of $X$ in $Y$. Without loss of generality, we may replace $Y$ by the normal bundle of $X$ in $Y$. This is because the exponential map associated to a $G$-invariant Riemannian metric intertwines the induced action of $G$ on a neighborhood of the zero section in the normal bundle with the $G$ action in a neighborhood of the submanifold in $Y$. Thus we may assume that we have two symplectic forms $\omega_{0}$ and $\omega_{1}$ on a vector bundle over $X$ and that $\omega_{1}-\omega_{0}$ is the zero form on the zero section. The homotopy $\varphi_{t}$ defined by radial contraction in the fiber, namely

$$
\phi_{t}(y)=(1-t) y
$$

satisfies $\phi_{0}=$ identity, $\phi_{1}(Y)=$ zero section, $\phi_{t}$ fixes the zero section, and $\phi_{t}$ is $G$-equivariant because $G$ acts by vector bundle maps. Now

$$
\begin{aligned}
-\left(\omega_{1}-\omega_{0}\right) & =\phi_{1}^{*}\left(\omega_{1}-\omega_{0}\right)-\left(\omega_{1}-\omega_{0}\right) \\
& =\int_{0}^{1} \frac{d}{d t} \phi_{t}^{*}\left(\omega_{1}-\omega_{0}\right) d t \\
& =\int_{0}^{1} \phi_{t}^{*}\left(\mathcal{L}_{\xi_{t}}\left(\omega_{1}-\omega_{0}\right)\right) d t \\
& \left.=\int_{0}^{1} \phi_{t}^{*}\left(d\left(\xi_{t}\right\lrcorner\left(\omega_{1}-\omega_{0}\right)\right)\right) d t \\
& \left.=d \int_{0}^{1} \phi_{t}^{*}\left(\xi_{t}\right\lrcorner\left(\omega_{1}-\omega_{0}\right)\right) d t .
\end{aligned}
$$

Therefore set

$$
\left.\zeta(y)=-\int_{0}^{1} \phi_{t}^{*}\left(\xi_{t}(y)\right\lrcorner\left(\omega_{1}-\omega_{0}\right)(y)\right) d t .
$$


Since $\phi_{t}$ is $G$-equivariant and $\xi_{t}, \omega_{1}$ and $\omega_{0}$ are $G$-invariant, we conclude that $\zeta$ is $G$-invariant and since $\omega_{1}-\omega_{0}$ vanishes on the zero section, so does $\zeta$. This concludes the proof of the Darboux theorem.

It remains to prove Theorem 9 on the uniqueness of constant rank embeddings.

Theorem 9 (Uniqueness of constant rank embeddings). Let $(P, \tau)$ and $\left(P^{\prime}, \tau^{\prime}\right)$ be two symplectic manifolds. Suppose $i: X \rightarrow(P, \tau)$ and $i^{\prime}$ : $X \rightarrow\left(P^{\prime}, \tau^{\prime}\right)$ are two constant rank embeddings with isomorphic symplectic normal bundles such that $i^{*} \tau=\left(i^{\prime}\right)^{*} \tau^{\prime}$. Then there exist neighborhoods $U$ of $i(X)$ in $P$ and $U^{\prime}$ of $i^{\prime}(X)$ in $P^{\prime}$ and a diffeomorphism $\phi: U \rightarrow U^{\prime}$ such that $\phi \circ i=i^{\prime}$ and $\phi^{*} \tau^{\prime}=\tau$.

Furthermore, if $G$ is a Lie group that acts properly on $X, P$ and $P^{\prime}$, preserves the forms $\tau$ and $\tau^{\prime}$ and if the embeddings $i$ and $i^{\prime}$ are $G$ equivariant, then $U$ and $U^{\prime}$ can be chosen to be $G$ invariant and $\phi$ to be $G$ equivariant.

Proof. The relative Darboux theorem says that a neighborhood of a submanifold $X$ in a symplectic manifold $(P, \tau)$ is symplectically determined by the symplectic vector bundle $T_{X} P$.

Now suppose $i: X \hookrightarrow(P, \tau)$ is a constant rank embedding. Then $\nu=$ $T X^{\tau} \cap T X$, the null distribution of the pull-back $i^{*} \tau$, is a vector bundle. We have also two symplectic vector bundles: the symplectic normal bundle of the embedding $N=T X^{\tau} / \nu$ and the bundle $E=T X / \nu$. We claim that, as a symplectic vector bundle, the bundle $T_{X} P$ is isomorphic to the direct sum $E \oplus N \oplus\left(\nu \oplus \nu^{*}\right)$ where the symplectic form $\omega_{\nu \oplus \nu^{*}}$ on $\nu \oplus \nu^{*}$ is given by

$$
\omega_{\nu \oplus \nu^{*}}(l, v)=l(v)
$$

(here $l \in \nu_{x}^{*}$ and $v \in \nu_{x}$ ). The claim would establish the theorem. Indeed, if $i^{\prime}: X \rightarrow\left(P^{\prime}, \tau^{\prime}\right)$ is another embedding with $\left(i^{\prime}\right)^{*} \tau^{\prime}=i^{*} \tau$ and $N^{\prime}=N$ then, according to the claim, $T_{X} P^{\prime} \simeq T_{X} P$ as symplectic vector bundles and the result follows from the Darboux theorem.

To prove the claim choose an almost complex structure $J$ adapted to $\tau$, i.e., choose $J$ such that $\tau(J \cdot, J \cdot)=\tau(\cdot, \cdot)$ and $g(\cdot, \cdot)=\tau(\cdot, J \cdot)$ is a positive definite metric. Then for any $v \in T X$ and any $w \in J \nu$ (with the same base point) we have

$$
g(v, w)=g(v, J(-J w))=\tau(v,-J w)=0
$$

since $J w \in \nu=T X^{\tau}$. So the bundle $J \nu$ lies in the metric perpendicular $T X^{g}$ of $T X$ and therefore $J \nu \cap T X=0$. It follows that the map $\phi: J \nu \rightarrow \nu^{*}$ 
defined as the composition of $\tau^{\#}: J \nu \rightarrow T_{X}^{*} P$ and of the natural projection $T_{X}^{*} P \rightarrow \nu^{*}$ is an isomorphism. Also for any $v \in J \nu$ and any $w \in \nu$ we have

$$
\tau(v, w)=\left\langle\tau^{\#} v, w\right\rangle=\langle\phi(v), w\rangle .
$$

Therefore $\phi \times i d: J \nu \oplus \nu \rightarrow \nu^{*} \oplus \nu$ is a isomorphism of symplectic vector bundles.

The natural map $\nu^{g} \cap T X \rightarrow T X / \nu=E$ is also a symplectic isomorphism. We conclude that $T X \oplus J \nu$ is a symplectic subbundle of $T_{X} P$ isomorphic to $E \oplus\left(\nu^{*} \oplus \nu\right)$. Finally observe that the symplectic perpendicular $T X^{\tau}$ of $T X$ satisfies $T X^{\tau}=(T X \oplus J \nu)^{\tau} \oplus \nu$. It follows that

$$
T_{X} P \simeq E \oplus N \oplus\left(\nu \oplus \nu^{*}\right)
$$

as symplectic vector bundles.

Note that if there is a group $G$ acting properly on our data, we can make the isomorphism (5) above $G$ equivariant by choosing a $G$ equivariant almost complex structure.

\section{References}

[ACG] J.M. Arms, R. Cushman and M. Gotay, A universal reduction procedure for Hamiltonian group actions, in The geometry of Hamiltonian systems, proceedings of a workshop held June 5-16, 1989, T. Ratiu, ed., New York, Springer-Verlag, 1991.

[AGJ] J.M. Arms, M. Gotay and G. Jennings, Geometric and algebraic reduction for singular momentum maps, Adv. in Math., 79 (1990), 43-103.

[AMM] J.M. Arms, J.E. Marsden and V. Moncrief, Symmetry and bifurcations of momentum mappings, Comm. Math. Phys., 78 (1981), 455-478.

[AG] V.I. Arnold and A.B. Givental, Symplectic geometry, in Dynamical systems IV, V.I. Arnold and S.P. Novikov, eds., Berlin, New York, Springer-Verlag, 1990.

[B] E. Bierstone, The structure of orbit spaces and the singularities of equivariant mappings, Monografias de matemática, 35, Instituto de Matemática Pura e Aplicada, Rio de Janeiro, 1980.

[CS] R. Cushman and R. Sjamaar, On singular reduction of Hamiltonian spaces, in Symmetric geometry and mathematical physics, actes du colloque en l'honneur de JeanMarie Souriau, P. Donato et al., eds., Boston, Birkhauser, 1991.

[GLS] V. Guillemin, E. Lerman and S. Sternberg, Symplectic fibrations and multiplicity diagrams, Cambridge University Press, 1995.

[GS1] V. Guillemin and S. Sternberg, A normal form for the moment map, in Differential geometric methods in mathematical physics, S. Sternberg, ed., Dordrecht, D. Reidel Publishing Company, 1984.

[GS2] _ Symplectic techniques in Physics, Cambridge University Press, 1990 (second reprint with corrections). 
[LMS] E. Lerman, R. Montgomery and R. Sjamaar, Examples of singular reduction, in Symplectic geometry, D.A. Salamon, ed., Cambridge University Press, 1993.

[Ma1] C.-M. Marle, Modèle d'action hamiltonienne d'un groupe de Lie sur une variété symplectique, Rendiconti del Seminario Matematico, Università e Politechnico, Torino, 43 (1985), 227-251.

[Ma2] _ Sous-veriété de rang constant d'une veriété symplectiques, Astérisque, 107108 (1983), 69-86.

[MW] J. Marsden and A. Weinstein, Reduction of symplectic manifolds with symmetry, Rep. Math. Phys., 5 (1974), 121-130.

[Me] K.R. Meyer, Symmetries and integrals in mechanics, in Dynamical systems; proceedings of Salvador Symposium on Dynamical Systems (1971, University of Bahia), M.M. Peixoto, ed., New York, Academic Press, 1973.

[O] M. Otto, A reduction scheme for phase spaces with almost Kähler symmetry. Regularity results for momentum level sets, J. Geom. Phys., 4 (1987), 101-118.

[P] L. Pukanszky, Unitary representations of solvable groups, Ann. Sci. Ecole Normale Sup., 4 (1971), 457-608.

[Sch] G.W. Schwarz, Smooth functions invariant under the action of a compact Lie group, Topology, 14 (1975), 63-68.

[SL] R. Sjamaar and E. Lerman, Stratified symplectic spaces and reduction, Ann. Math., 134 (1991), 375-422.

[SSB] J. Śniatycki, G. Schwartz and L. Bates, Yang-Mills and Dirac fields in a bag, constraints and reduction, Comm. Math. Phys., 176 (1996), 95-115.

Received May 25, 1995 and revised June 17, 1996. The second author was supported by an NSF postdoctoral fellowship.

\author{
UNIVERSITY OF CALGARY \\ Calgary, Alberta \\ CANADA T2N $1 \mathrm{~N} 4$ \\ AND \\ UNIVERSITY OF ILLINOIS \\ URBANA, IL 61801
}

